\title{
Knowledge, Attitude and Practice of Medical Students of Sulaimani Regarding Modern Lifestyle and Its Effect on Health
}

\author{
Bushra M. Ali \\ Family \& Community Medicine Department \\ College of Medicine \\ University of Sulaimani \\ Sulaimani, Iraq \\ bmakfb.2002@yahoo.com
}

\author{
Shajwan Akram \\ College of Medicine \\ University of Sulaimani \\ Sulaimani, Iraq \\ shajwanakram10@gmail.com \\ Lezan Baxtiar \\ College of Medicine \\ University of Sulaimani \\ Sulaimani, Iraq \\ lezanbaxtiar@gmail.com \\ Shad Nawzad \\ College of Medicine \\ University of Sulaimani \\ Sulaimani, Iraq \\ shadnawzad919@gmail.com
}

\author{
Saman Mohammed \\ Family \& Community Medicine Department \\ College of Medicine \\ University of Sulaimani \\ Sulaimani, Iraq \\ dr.saman_am@yahoo.com \\ Saya Salar \\ College of Medicine \\ University of Sulaimani \\ Sulaimani, Iraq \\ saya.maziz330@gmail.com \\ Lawen Ismail \\ College of Medicine \\ University of Sulaimani \\ Sulaimani, Iraq \\ lawenismai144@gmail.com
}

\begin{tabular}{l} 
Article Info \\
\hline Volume 5 - Special Issue: $4^{\text {th }}$ \\
International Conference on the Health \\
and Medical Science : Medical \\
Researches Improve Life Quality \\
(ICHMS 2020) \\
DOI: \\
10.24017/science.2020.ICHMS2020.6 \\
Article history: \\
Received : 22 September 2020 \\
Accepted : 02 October 2020 \\
\hline
\end{tabular}

\begin{abstract}
The objectives of this study are to assess level of knowledge, attitude and practice of medical students about modern lifestyle and its impact upon health, in association with different colleges. A cross sectional study was conducted among 420 students, in a convenient sampling way, Data collected from $18^{\text {th }}$ to $22^{\text {nd }}$ of September 2019 in a self-administrated manner from four different medical colleges (Medicine, Dentistry, Pharmacy and Veterinary.) in university of Sulaimani. Data entered on Excel sheets and analyzed with SPSS program version 22. Chi square was used to get the
\end{abstract}


Keywords:

Modern life style, Medical students, Sulamani. Iraq association $P$ value equal and less than (0.05) regarded statistically significant. Most of the students (82.1\%) had heard about a modern lifestyle with highest percentage of participants (90.5\%) from college of medicine, and (69.3\%) knew that technology has a major role in creating a modern lifestyle, with a significant $P$ value of (0.001). Nearly half of the students (54.5\%) believed to be living a modern lifestyle and most of them (66.2\%) were from college of dentistry. About (49.5\%) of students believed that financial status can sometimes put limits on lifestyle choice with most of them (52.7\%) being from college of dentistry with $p$ value of (0.001). Majority of students (995\%) used social media with most of them (97.4\%) being from college of medicine. Nearly (66\%) of participants didn't exercise and highest percentage (78.1\%) from college of pharmacy with a significant ( $P$ value 0.007). Participants in general had good knowledge and positive attitude regarding the topic, but they had a negative practice with managing stress, time and depression and had negative habits regarding exercise and having healthy diet. In addition college of medicine had highest knowledge and attitude among the four medical colleges, but regarding the practice college of pharmacy was the best.

Copyright $(\mathrm{C} 2020$ Kurdistan Journal of Applied Research.

All rights reserved.

\section{INTRODUCTION}

Life style is defined as way of living of individuals, families, and societies, which they manifest in coping with their physical, and psychological, social, and economical environment on a daily basis...this definition explains that a particular lifestyle of an individual has a great impact on activities, interests, their opinions and ambitions, as lifestyle can be treated as an indicator of social attitudes and of the ideologies that are functioning in the social space [1]. On the other hand, life style itself is tremendously affected by multiple factors including family, culture, social class, the individuals instincts, technology, and social media. In the olden days, life was natural, slow, and somehow difficult at time but nowadays in modern times, life is fast paced, comfortable, readily made but however, is stressful. Modern life style slowly crept in with changing times [2]. Modern lifestyle offers important information about nutrition, physical activity, stress control, medical services, physical/mental health, social support, education, technology, and our chief goals. Regarding diet and nutrition, an important aspect of modern lifestyle is the change in food habit. In most homes traditional simple food has given way to fast food, which is high in calories, especially children and young adults are too attracted to fried and oily food that now is easily available. Also eating habits, such as eating while watching TV(snacking between meals and munching when there's no work ,all has contributed to life style diseases. these caused children to adults put on weight which has become a very common problem that has exposed us to some dangerous health hazards like high blood pressure, diabetes and obesity, these conditions which were earlier seen in people past fifty years are now seen in younger age groups .and they've become a common lifestyle 
diseases and are causes of worry all over the world. [2] It goes without saying that technology had a major role in building modern society and lifestyle, and there's absolutely no doubt that over the years technology has been responsible for creating amazingly useful resources which put all the information we need at our fingertips. The development of technology has led to so many mind-blowing discoveries, better facilities, and better luxuries, but at the same has dramatically changed our daily lives. Various highly-developed gadgets, connected to the Internet, have changed the way we communicate, use humor, study, work, shop, play, and behave. [3] Previously children were going out to play, but nowadays seeing children playing and sweat it out is a rare sight, with everyone glued to the TV or play video games or on the internet for hours. This along with growing competition among parents and facilities, kids are forced to attend many hobby classes and tuitions, all of which leaves less time for playing and having outdoor physical activities that has impact on the health. Same goes for adults, with advancements in technology, everything from social gatherings, variety of facilities available for transportation, to environment at work places and many more changed from what they were in the past .and with all this change it's difficult to find time for physical activities. All of these factors created a sedentary life style which has become a lead to many diseases. [2] In addition, one of the bases of healthy life is sleep. Sleep cannot be apart from life. And nowadays sleep disorders have several social, psychological, economical and health consequences. Lifestyle can has effect on sleep which has clear influence on mental and physical health. [3] The other aspect of modern lifestyle is level of medical services that has much progressed compared to the past regarding primary care and prevention, providing health insurances, vaccinations, many facilities for disease screening and new treatment for diseases and even cure for some. These are a lot of things that nobody would have thought were possible a few years back but now are being practiced and they're actually saving lives. Lifestyle describes a complex behavioral strategies and routines, attitudes and values, and with each day advancements we create a modern way of living that has impact on us in almost every way. The role of health in terms of modern lifestyle is very well recognized nowadays. [4] Evaluations of healthy lifestyle among Greek university students demonstrated that there was a positive correlation between medical student's healthy behavior and their quality of life. Also several studies have been conducted in Iran indicating that the majority of medical students did not have appropriate physical activity nor sleep pattern. [5]. The aims of this study are to estimate knowledge, attitude, and practice of medical students of university of Sulaimani regarding the modern lifestyle and effects on health and to estimate association between different colleges and their knowledge, attitude, practice about modern lifestyle.

\section{METHODS AND MATERIALS}

For this research, a cross sectional study was conducted. The study was conducted in university of Sulaimani, faculty of medical sciences, included four different medical colleges: colleges of medicine, dentistry, pharmacy and veterinary. From 18th to 29th of September 2019. A sample of 420 students participated from all colleges and all stages of each college was conducted in a convenient way. A self-administered questionnaire was prepared consisted of four sections:8 questions about Socio-demographic, 9 questions about knowledge of students regarding lifestyle, another 9 about attitude and 9 about practice. All making a total of 35 questions. A pilot study has been done and study was adjusted accordingly with help of a few medical colleagues within same colleges in which study was conducted. Data collection was in both online google forms (340) and hard copies (80) with keeping total privacy and participants and reassuring their agreement to fill out. Data were entered on excel sheets (Microsoft Excel 2010), all analyzed with SPSS program (Version 22).Tables and graphics were used to present data in a descriptive statistical way. Chi square: used to test the association between variables and $\mathrm{P}$ value was accounted as probability of statistics with value of 0.05 and less taken as significant.

For Ethical considerations, official permission was taken from college authority for distribution of hard copies, and informed consent was taken from all participants for both 
online and hard copy. All students were informed that their participation is completely voluntary and all the collected information was kept confidential.

\section{RESULTS}

(Table 1) Shows socio-demographic status that majority of participants were single (93.3\%) and female $(60.2 \%)$, they were mostly from inside city $(68.3 \%)$ and majority had a moderate financial status $(77.9 \%)$, with the highest percentage of participants being in college of medicine $(55 \%)$.

Table1: Socio-demographic data of the sample.

\begin{tabular}{ccc}
\hline SDD & Number & Percentage \% \\
Gender & & \\
Female & 253 & $\mathbf{( 6 0 . 2 )}$ \\
Male & 167 & $\mathbf{( 3 9 . 8 )}$ \\
\hline Residency & & \\
Inside city & 287 & $\mathbf{( 6 8 . 3 )}$ \\
Outside city & 133 & $\mathbf{( 3 1 . 7 )}$ \\
\hline College & & \\
Medicine & 231 & $\mathbf{( 5 5 )}$ \\
Dentistry & 74 & $\mathbf{( 1 7 . 6 )}$ \\
Pharmacy & 64 & $\mathbf{( 1 5 . 2 )}$ \\
Veterinary & 51 & $\mathbf{( 1 2 . 1 )}$ \\
\hline Marital status & & \\
Single & 392 & $\mathbf{( 9 3 . 3 )}$ \\
Married & 26 & $\mathbf{( 6 . 2 )}$ \\
Divorced/widow & 2 & $\mathbf{( 0 . 5 )}$ \\
\hline Socioeconomic & & \\
status & 39 & $\mathbf{( 9 . 3 )}$ \\
Low & 327 & $\mathbf{( 7 7 . 9 )}$ \\
Moderate & 54 & $\mathbf{( 1 2 . 9 )}$ \\
High & & \\
\hline Total & 420 & $\mathbf{( 1 0 0 )}$ \\
\hline
\end{tabular}

(Figure 1) shows distribution of participants according to BMI group. It shows that the majority of the participants (75.95) were in normal Body Mass Index group.

\section{BMI Group}

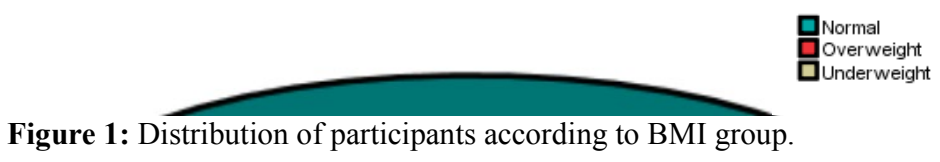

Kurdistan Journal of Applied Research $\mid 4^{\text {th }}$ International Conference on the Health and Medical Science: Medical Researches Improve Life Quality (ICHMS 2020) | 52 
(Table 2) shows the association between different colleges and their knowledge regarding lifestyle, most of the participants $(82.1 \%)$ had heard of modern lifestyle with highest percentage from college of medicine take highest percentage $(90.5 \%)$, proven by $\mathrm{P}$ value of (0.001) that states a significant and strong association. And nearly half of these students had heard about it from the internet (49.3\%). And highest percentage (77.4\%) of participants knew lifestyle can affect mental health and were mostly from college of Medicine (87\%) and there is a significant association between the colleges and their answer $(\mathrm{P}$ value $=0.001)$. And again there's a significant association between colleges and their knowledge regarding the effect of physical health that most of participant $(78.3 \%$ ) had knowledge and majority were from college of medicine $(90.5 \%)$ with $\mathrm{P}$ value significance of (0.001) stating association. And most of participants $(73.3 \%)$ took lifestyle as a factor that can affect academic life ,and among them college of medicine predominated and (84\%) of "yes" answers which was dominant answer were from them, with a $\mathrm{P}$ value of (0.001) stating significant association. Regarding the effect of technology on lifestyle the highest percentage $(69.3 \%)$ of responders said yes and most of them were from college of medicine (78.8\%) with correct answer, A P value of (0.001) states significant associations between the college of participants and their answer to the question. And most of participants $(68.3 \%)$ regarded regular medical checkups being a part of modern lifestyle, with highest percentage were from college of Medicine (78.8\%),P value was equal to (0.001) showing clear and significant association similarly, Diabetes/hypertension as a wrong answer was chosen and majority who answered this way were from college of medicine $(51.5 \%)$ a $P$ value of $(0.001)$ shows significant association.

Table 2: Association between different colleges students and their knowledge regarding modern lifestyle

\begin{tabular}{|c|c|c|c|c|c|c|}
\hline Questions & $\begin{array}{l}\text { Medicine } \\
\text { No. } / \%\end{array}$ & $\begin{array}{c}\text { Dentistry } \\
\text { No. } / \%\end{array}$ & $\begin{array}{c}\text { Pharmacy } \\
\text { No. } / \%\end{array}$ & $\begin{array}{c}\text { Veterinary } \\
\text { No. } / \%\end{array}$ & $\begin{array}{c}\text { Total } \\
\text { No. } / \%\end{array}$ & P. value \\
\hline $\begin{array}{l}\text { Have you heard } \\
\text { of modern } \\
\text { lifestyle? } \\
\text { Yes } \\
\text { No }\end{array}$ & $\begin{array}{c}209(90.47) \\
22(9.52)\end{array}$ & $\begin{array}{l}52(70.3) \\
22(29.7)\end{array}$ & $\begin{array}{l}44(68.8) \\
20(31.3)\end{array}$ & $\begin{array}{l}40(78.4) \\
11(21.6)\end{array}$ & $\begin{array}{c}345(82.1) \\
75(17.9)\end{array}$ & 0.001 \\
\hline $\begin{array}{l}\text { From where did } \\
\text { you hear about } \\
\text { it? } \\
\text { People } \\
\text { Internet } \\
\text { Book } \\
\text { College } \\
\text { Academic life } \\
\text { Those who didn't } \\
\text { hear about it }\end{array}$ & $\begin{array}{c}40(17.3) \\
140(60.6) \\
10(4.3) \\
19(8.2) \\
0(0.0) \\
22(9.52)\end{array}$ & $\begin{array}{c}12(16.2) \\
23(31.1) \\
5(16.2) \\
12(6.8) \\
0(0.0) \\
22(29.7)\end{array}$ & $\begin{array}{c}9(14.1) \\
23(35.9) \\
3(14.1) \\
9(4.7) \\
0(0.0) \\
20(31.3)\end{array}$ & $\begin{array}{c}10(19.6) \\
21(41.2) \\
4(7.8) \\
4(7.8) \\
1(0.2) \\
11(21.6)\end{array}$ & $\begin{array}{c}71(16.9) \\
207(49.3) \\
22(5.2) \\
45(10.7) \\
1(0.2) \\
75(17.9)\end{array}$ & 0.001 \\
\hline $\begin{array}{l}\text { lifestyle can } \\
\text { affect mental } \\
\text { health? } \\
\text { Yes } \\
\text { No } \\
\text { I don't know }\end{array}$ & $\begin{array}{l}201(87) \\
12(5.2) \\
18(7.8)\end{array}$ & $\begin{array}{c}47(63.5) \\
17(23) \\
10(13.5)\end{array}$ & $\begin{array}{l}45(70.3) \\
9(14.1) \\
10(15.6)\end{array}$ & $\begin{array}{c}32(62.7) \\
9(17.6) \\
10(19.6)\end{array}$ & $\begin{array}{r}325(77.4) \\
47(11.2) \\
48(11.4)\end{array}$ & 0.001 \\
\hline $\begin{array}{l}\text { lifestyle can } \\
\text { affect physical } \\
\text { health? } \\
\text { Yes } \\
\text { No } \\
\text { I don't know }\end{array}$ & $\begin{array}{c}209(90.5) \\
15(6.5) \\
7(3)\end{array}$ & $\begin{array}{c}46(62.2) \\
11(14.9) \\
17(23)\end{array}$ & $\begin{array}{c}44(68.8) \\
7(10.9) \\
13(20.3)\end{array}$ & $\begin{array}{l}30(58.8) \\
10(19.6) \\
11(21.6)\end{array}$ & $\begin{array}{c}329(78.3) \\
43(10.2) \\
48(11.4)\end{array}$ & 0.001 \\
\hline
\end{tabular}

Kurdistan Journal of Applied Research $\mid 4^{\text {th }}$ International Conference on the Health and Medical Science: Medical Researches Improve Life Quality (ICHMS 2020) | 53 


\begin{tabular}{|c|c|c|c|c|c|c|}
\hline $\begin{array}{c}\text { performance? } \\
\text { Yes } \\
\text { No } \\
\text { I don't know } \\
\end{array}$ & $\begin{array}{l}194(84) \\
18(7.8) \\
19(8.2)\end{array}$ & $\begin{array}{l}44(59.5) \\
11(14.9) \\
19(25.7)\end{array}$ & $\begin{array}{l}39(60.9) \\
11(17.2) \\
14(21.9)\end{array}$ & $\begin{array}{c}31(60.8) \\
9(17.6) \\
11(21.6)\end{array}$ & $\begin{array}{c}308(73.3) \\
49(11.7) \\
63(15)\end{array}$ & 0.001 \\
\hline $\begin{array}{c}\text { Technology has a } \\
\text { major role in } \\
\text { modern lifestyle? } \\
\text { Yes } \\
\text { No } \\
\text { I don't know } \\
\end{array}$ & $\begin{array}{l}182(78.8) \\
21(9.1) \\
28(12.1)\end{array}$ & $\begin{array}{c}45(60.8) \\
8(10.8) \\
21(28.4)\end{array}$ & $\begin{array}{l}31(48.4) \\
14(21.9) \\
19(29.7)\end{array}$ & $\begin{array}{c}33(64.7) \\
3(5.9) \\
15(29.4)\end{array}$ & $\begin{array}{c}291(69.3) \\
46(11) \\
83(19.7)\end{array}$ & 0.001 \\
\hline $\begin{array}{l}\text { Healthy diet and } \\
\text { regular medical } \\
\text { checkup is part } \\
\text { of a healthy } \\
\text { modern lifestyle? } \\
\text { Yes } \\
\text { No } \\
\text { I don't know } \\
\end{array}$ & $\begin{array}{l}182(78.8) \\
16(6.9) \\
33(14.3) \\
\end{array}$ & $\begin{array}{c}37(50) \\
14(18.9) \\
23(31.1)\end{array}$ & $\begin{array}{l}38(59.4) \\
7(10.9) \\
19(29.7)\end{array}$ & $\begin{array}{c}30(58.8) \\
6(11.8) \\
15(29.4)\end{array}$ & $\begin{array}{r}287(68.3) \\
43(10.2) \\
90(21.5)\end{array}$ & 0.001 \\
\hline Total & $231(100)$ & $74(100)$ & $64(100)$ & $51(100)$ & $420(100)$ & \\
\hline
\end{tabular}

(Figure 2) shows the association among college students regarding knowledge about impact of lifestyle on nutritional deficiencies or illnesses. Most of participants(68.1\%) knew that illness and deficiency can be related to certain lifestyle, college of medicine students took the highest percentage $(81.8 \%)$ regarding knowledge and $p$ value of $(0.001)$ states significant association.

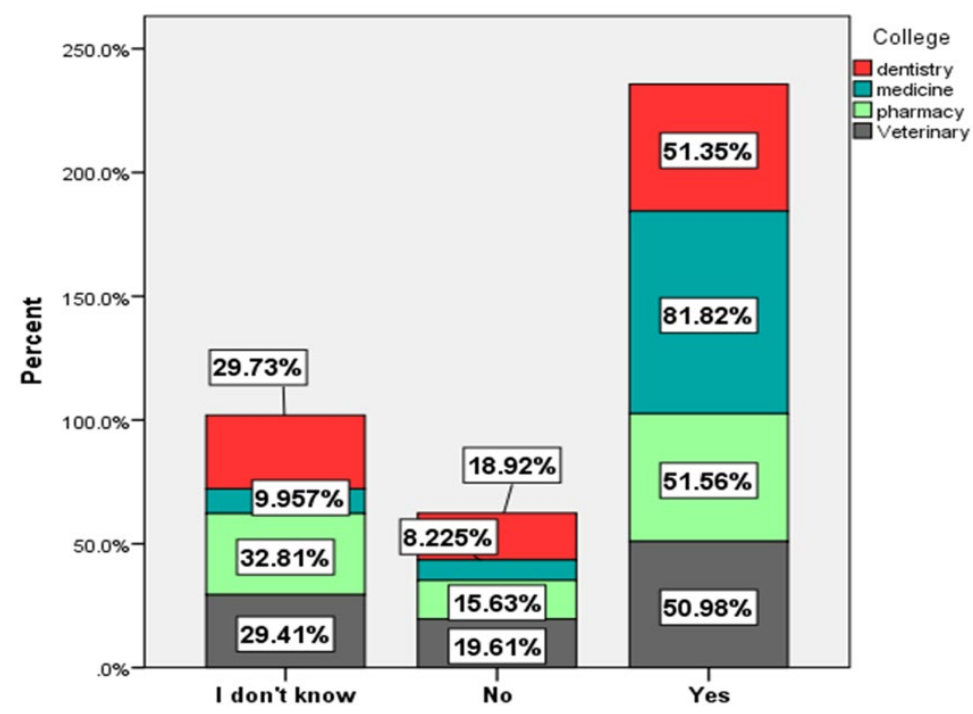

Figure 2: Association among college students regarding their knowledge about impact of lifestyle on nutritional deficiencies or illnesses

(Table 3) shows association between colleges and the attitude of students regarding modern lifestyle clarifying that about $(54.5 \%)$ of participants think they live a modern lifestyle, and highest percentage $(66.2 \%)$ are from college of Dentistry , and a $\mathrm{P}$ value of $(0.078)$ does not state the association (meaning that there is no significant association between the college and their answer to the question. And about $(64.3 \%)$ of participants only sometimes were willing 
to change their lifestyle inspired by other people, and highest percentage $(73.6 \%)$ were from college of medicine and $\mathrm{P}$ value of (0.001) shows significant association. Highest percentage of respondents $(65.2 \%)$ thought keeping up with certain lifestyle can sometimes be stressful, and highest percentage of them(70.6\%) were from college of medicine, $\mathrm{P}$ value of $(0.001)$ states significant association. And most of participants $(66 \%)$ think people sometimes pretended to have a certain lifestyle to attract people and most of them $(75.3 \%)$ were from college of medicine, $\mathrm{P}$ value of (0.001) states significant association with the college. Nearly half of students $(49.5 \%)$ thought financial status can sometimes put limits on living a certain lifestyle, and most of those (57.6) were from college of medicine and $\mathrm{P}$ value of (0.001) shows significant association with the college. And regarding religion, nearly half $(45.5 \%)$ believe that it can force a certain lifestyle, with highest percentage being from college of veterinary $(52.9 \%)$ a $\mathrm{P}$ value of (0.019) rules out any significant association with this factor. Nearly half of the students (46.4\%) that geographical area always affects choosing a lifestyle with highest percentage being from college of pharmacy $(51.6 \%)$ with $p$ value $=0.078$ indicating it's not significant. And only about $(64.5 \%)$ think that diet restrictions due to lifestyle can sometimes lead to illness and nutrition deficiency with highest percentage being from college of pharmacy $(73.4 \%)$ and $(\mathrm{P}$ value $=0.113$, no significant association is stated.

Table 3: Association between different colleges students and their attitude regarding modern lifestyle.

\begin{tabular}{|c|c|c|c|c|c|c|}
\hline Questions/attitude & $\begin{array}{c}\text { Medicine } \\
\text { No. } / \%\end{array}$ & $\begin{array}{c}\text { Dentistry } \\
\text { No. } / \% \\
\end{array}$ & $\begin{array}{c}\text { Pharmacy } \\
\text { No./\% }\end{array}$ & $\begin{array}{c}\text { Veterinary } \\
\text { No. } / \% \\
\end{array}$ & $\begin{array}{c}\text { Total } \\
\text { No. } / \% \\
\end{array}$ & P. value \\
\hline $\begin{array}{c}\text { In your opinion } \\
\text { are you living a } \\
\text { modern lifestyle? } \\
\qquad \begin{array}{c}\text { Yes } \\
\text { No }\end{array} \\
\end{array}$ & $\begin{array}{l}117(50.6) \\
114(49.4)\end{array}$ & $\begin{array}{l}49(66.2) \\
25(33.8)\end{array}$ & $\begin{array}{l}32(50) \\
32(50)\end{array}$ & $\begin{array}{l}31(60.8) \\
20(39.2)\end{array}$ & $\begin{array}{l}229(54.5) \\
191(45.5)\end{array}$ & 0.078 \\
\hline $\begin{array}{l}\text { You are willing to } \\
\text { make changes in } \\
\text { your life inspired } \\
\text { by people around } \\
\text { you? } \\
\text { Always } \\
\text { Sometimes } \\
\text { Never/rarely }\end{array}$ & $\begin{array}{c}27(11.7) \\
170(73.6) \\
34(14.7)\end{array}$ & $\begin{array}{l}11(14.9) \\
34(45.9) \\
29(39.2)\end{array}$ & $\begin{array}{c}9(14.1) \\
29(45.3) \\
26(40.6)\end{array}$ & $\begin{array}{c}6(11.8) \\
37(72.5) \\
8(15.7)\end{array}$ & $\begin{array}{c}53(12.6) \\
270(64.3) \\
97(23.1)\end{array}$ & 0.001 \\
\hline $\begin{array}{l}\text { Do you think that } \\
\text { keeping up with a } \\
\text { certain lifestyle } \\
\text { can be stressful? } \\
\text { Always } \\
\text { Sometimes } \\
\text { Never/rarely }\end{array}$ & $\begin{array}{c}47(20.3) \\
163(70.6) \\
21(9.1)\end{array}$ & $\begin{array}{l}18(24.3) \\
43(58.1) \\
13(17.6)\end{array}$ & $\begin{array}{l}14(21.9) \\
35(54.7) \\
15(23.4)\end{array}$ & $\begin{array}{c}11(21.6) \\
33(64.7) \\
7(13.7)\end{array}$ & $\begin{array}{c}90(21.4) \\
274(65.2) \\
56(13.3)\end{array}$ & 0.059 \\
\hline $\begin{array}{l}\text { Do you think } \\
\text { people may } \\
\text { pretend to have a } \\
\text { certain lifestyle to } \\
\text { attract others? } \\
\text { Always } \\
\text { Sometimes } \\
\text { Never/rarely }\end{array}$ & $\begin{array}{c}34(14.7) \\
174(75.3) \\
23(10)\end{array}$ & $\begin{array}{l}25(33.8) \\
38(51.4) \\
11(14.9)\end{array}$ & $\begin{array}{l}20(31.3) \\
33(51.6) \\
11(17.2)\end{array}$ & $\begin{array}{c}12(23.5) \\
32(62.7) \\
7(13.7)\end{array}$ & $\begin{array}{l}91(21.7) \\
277(66) \\
52(12.4)\end{array}$ & 0.001 \\
\hline $\begin{array}{l}\text { Do you think } \\
\text { financial status } \\
\text { can put limits on } \\
\text { living a certain } \\
\text { lifestyle? } \\
\text { Always } \\
\text { Sometimes } \\
\text { Never/rarely } \\
\text { Do you think you }\end{array}$ & $\begin{array}{c}79(34.2) \\
133(57.6) \\
19(8.2)\end{array}$ & $\begin{array}{c}39(52.7) \\
29(39.2) \\
6(8.1)\end{array}$ & $\begin{array}{l}27(42.2) \\
23(35.9) \\
14(21.9)\end{array}$ & $\begin{array}{c}19(37.3) \\
23(45.1) \\
9(17.6)\end{array}$ & $\begin{array}{c}164(39) \\
208(49.5) \\
48(11.4)\end{array}$ & 0.001 \\
\hline
\end{tabular}

Kurdistan Journal of Applied Research $\mid 4^{\text {th }}$ International Conference on the Health and Medical Science: Medical Researches Improve Life Quality (ICHMS 2020) $\mid 55$ 


\begin{tabular}{|c|c|c|c|c|c|c|}
\hline $\begin{array}{l}\text { are forced to } \\
\text { follow a certain } \\
\text { lifestyle because of } \\
\text { you religion? }\end{array}$ & & & & & & 0.019 \\
\hline Always & $53(22.9)$ & $29(39.2)$ & $27(42.2)$ & $14(27.5)$ & $123(29.3)$ & \\
\hline Sometimes & $114(49.4)$ & $29(39.2)$ & $21(32.8)$ & $27(52.9)$ & $191(45.5)$ & \\
\hline Never/rarely & $64(27.7)$ & $16(21.6)$ & $16(25)$ & $10(19.6)$ & $106(25.2)$ & \\
\hline $\begin{array}{l}\text { Do you think } \\
\text { geographical area } \\
\text { affects choosing } \\
\text { modern lifestyle? }\end{array}$ & & & & & & 0.78 \\
\hline Always & $106(45.9)$ & $37(50)$ & $33(51.6)$ & $19(37.3)$ & $195(46.4)$ & \\
\hline Sometimes & $98(42.4)$ & $24(32.4)$ & $20(31.3)$ & $18(35.3)$ & $160(38.1)$ & \\
\hline Never/rarely & $27(11.7)$ & $13(17.6)$ & $11(17.2)$ & $14(27.5)$ & $65(15.5)$ & \\
\hline $\begin{array}{l}\text { Do you think any } \\
\text { diet restrictions } \\
\text { due to a certain } \\
\text { lifestyle changes } \\
\text { can lead to illness } \\
\text { and deficiency of } \\
\text { nutrition? }\end{array}$ & & & & & & 0.113 \\
\hline Always & $56(24.2)$ & $16(21.6)$ & $5(7.8)$ & $10(19.6)$ & $87(20.7)$ & \\
\hline Sometimes & $147(63.6)$ & $46(62.2)$ & $47(73.4)$ & $31(60.8)$ & $271(64.5)$ & \\
\hline Never/rarely & $28(12.2)$ & $12(16.2)$ & $12(18.8)$ & $10(19.6)$ & $62(14.8)$ & \\
\hline Total & $231(100)$ & $74(100)$ & $64(100)$ & $51(100)$ & $420(100)$ & \\
\hline
\end{tabular}

Figure 3: Shows the associations among colleges regarding attitude about lifestyle and their culture and it shows nearly half of participants (48.3\%) felt that sometimes culture can force a certain lifestyle with highest percentage from college of veterinary $(56.9 \%)$ with $(\mathrm{P}=0.001)$ showing significant association.

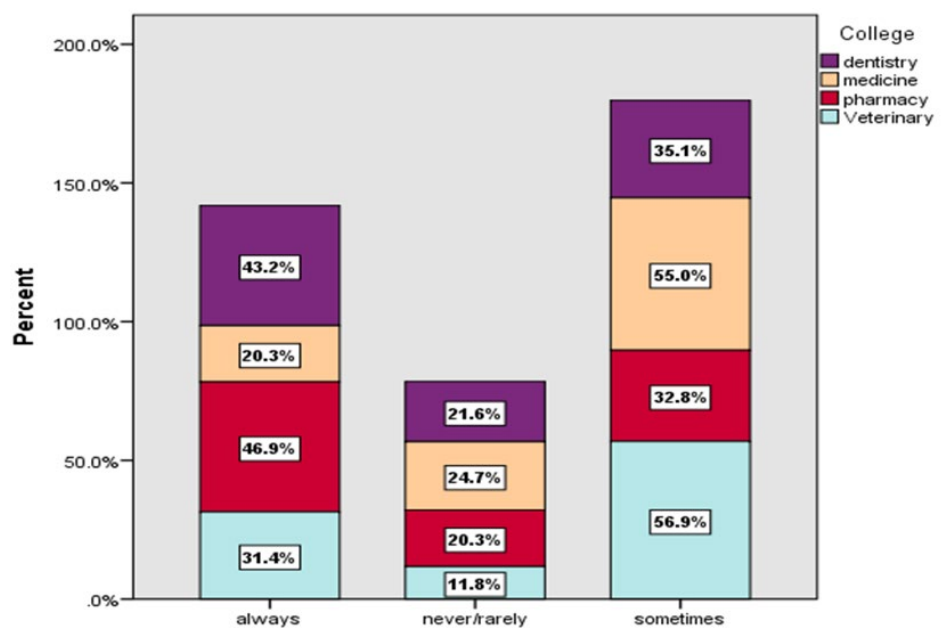

Figure 3: Associations among colleges students about if they feel that their culture force them to follow a certain lifestyle.

(Table 4) shows association between college of participants and their practice regarding lifestyle. Comparing current lifestyle to the past, most of participants $(59.8 \%)$ are feeling more stressed and majority is from college of medicine $(62.3 \%)$, and $\mathrm{P}$ value of $(0.485)$ rules out any significant association. Most of students (63.8\%) had difficulties arranging time due to lifestyle choice, and highest percentage being from college of medicine $(73.6 \%), \mathrm{P}$ value of 
(0.001) shows significant association. And majority of respondents (95\%) use social media as a part of their lifestyle, and highest percentage (97.4\%) was among medicine, $\mathrm{P}$ value of (0.077) shows that there's no significant association. Highest percentage of students $(70.9 \%)$ didn't playing video games, and most of them were from college of pharmacy $(75 \%), \mathrm{P}$ value of (0.457) rules out association. And data shows that most of participants $(66 \%)$ of students didn't exercise as a part of their lifestyle, highest percentage $(78.1 \%)$ were from college of pharmacy , $\mathrm{P}$ value of (0.007) states significant association. Half of Participants $(51.7 \%)$ never had depression because of not being able to have a certain lifestyle, with highest percentage being from college of pharmacy $(70.3 \%)$ ( $\mathrm{P}$ value=0.003). And healthy diet wasn't included as a part of lifestyle by most of responders $(59.3 \%)$, and they mostly (63.5) were from college of dentistry, $\mathrm{P}$ value of ( 0.681$)$ ruled out association, In addition most of them (58\%) didn't have health issues due to their diet ,and most of those who responded were pharmacy students $(60.9 \%)$ and $\mathrm{P}$ value of $(0.843)$ again rules out association.

Table 4: Association between different colleges students and their practice regarding modern lifestyle.

\begin{tabular}{|c|c|c|c|c|c|c|}
\hline $\begin{array}{c}\text { Questions/practic } \\
\mathrm{e} \\
\end{array}$ & $\begin{array}{c}\text { Medicine } \\
\text { No. } / \% \\
\end{array}$ & $\begin{array}{l}\text { Dentistry } \\
\text { No. } / \% \\
\end{array}$ & $\begin{array}{c}\text { Pharmacy } \\
\text { No./\% }\end{array}$ & $\begin{array}{c}\text { Veterinary } \\
\text { No. } / \%\end{array}$ & $\begin{array}{c}\text { Total } \\
\text { No./\% }\end{array}$ & $\begin{array}{c}\mathrm{P} . \\
\text { Value }\end{array}$ \\
\hline $\begin{array}{l}\text { Comparing your } \\
\text { current lifestyle } \\
\text { to your past, are } \\
\text { you more } \\
\text { stressed? } \\
\text { Yes } \\
\text { No } \\
\end{array}$ & $\begin{array}{c}144(62.3) \\
87(37.7)\end{array}$ & $\begin{array}{l}44(59.5) \\
30(40.5)\end{array}$ & $\begin{array}{l}33(51.6) \\
31(48.4\end{array}$ & $\begin{array}{l}30(58.8) \\
21(41.2)\end{array}$ & $\begin{array}{l}251(59.8) \\
169(40.2)\end{array}$ & 0.485 \\
\hline $\begin{array}{l}\text { Have you ever } \\
\text { had any } \\
\text { difficulties } \\
\text { arranging your } \\
\text { time? } \\
\quad \text { Yes } \\
\quad \text { No } \\
\quad \text { Sometimes }\end{array}$ & $\begin{array}{c}170(73.6) \\
52(22.5) \\
9(3.9)\end{array}$ & $\begin{array}{c}30(40.5) \\
43(58.1) \\
1(1.4)\end{array}$ & $\begin{array}{c}33(51.6) \\
31(48.4) \\
0(0)\end{array}$ & $\begin{array}{c}35(68.6) \\
15(29.4) \\
1(2)\end{array}$ & $\begin{array}{c}268(63.8) \\
141(33.6) \\
11(2.6)\end{array}$ & 0.001 \\
\hline $\begin{array}{l}\text { Do you use social } \\
\text { media? } \\
\qquad \begin{array}{c}\text { Yes } \\
\text { No }\end{array} \\
\end{array}$ & $\begin{array}{c}225(97.4) \\
6(2.6) \\
\end{array}$ & $\begin{array}{c}69(93.2) \\
5(6.8) \\
\end{array}$ & $\begin{array}{c}59(92.2) \\
5(7.8) \\
\end{array}$ & $\begin{array}{c}46(90.2) \\
5(9.8)\end{array}$ & $\begin{array}{c}399(95) \\
21(5)\end{array}$ & 0.077 \\
\hline $\begin{array}{c}\text { Do you play } \\
\text { video games? } \\
\text { Yes } \\
\text { No } \\
\end{array}$ & $\begin{array}{c}68(29.4) \\
163(70.6) \\
\end{array}$ & $\begin{array}{l}19(25.7) \\
55(74.3) \\
\end{array}$ & $\begin{array}{l}16(25) \\
48(75)\end{array}$ & $\begin{array}{l}19(37.3) \\
32(62.7) \\
\end{array}$ & $\begin{array}{l}122(29.1) \\
298(70.9) \\
\end{array}$ & 0.457 \\
\hline $\begin{array}{c}\text { Do you exercise? } \\
\text { Yes } \\
\text { No } \\
\end{array}$ & $\begin{array}{c}95(41.1) \\
136(58.9) \\
\end{array}$ & $\begin{array}{l}20(27) \\
54(73)\end{array}$ & $\begin{array}{l}14(21.9) \\
50(78.1)\end{array}$ & $\begin{array}{l}14(27.5) \\
37(72.5)\end{array}$ & $\begin{array}{l}143(34) \\
277(66) \\
\end{array}$ & 0.007 \\
\hline $\begin{array}{l}\text { Have you ever } \\
\text { had depression } \\
\text { because of not } \\
\text { being able to } \\
\text { have a lifestyle? } \\
\text { Yes } \\
\text { No } \\
\end{array}$ & $\begin{array}{c}126(54.5) \\
105(45.5)\end{array}$ & $\begin{array}{l}31(41.9) \\
43(58.1)\end{array}$ & $\begin{array}{l}19(29.7) \\
45(70.3)\end{array}$ & $\begin{array}{l}27(52.9) \\
24(47.1)\end{array}$ & $\begin{array}{l}203(48.3) \\
217(51.7) \\
\end{array}$ & 0.003 \\
\hline $\begin{array}{c}\text { Are you on a } \\
\text { healthy diet? } \\
\text { Yes } \\
\text { No } \\
\end{array}$ & $\begin{array}{l}100(43.3) \\
131(56.7) \\
\end{array}$ & $\begin{array}{l}27(36.5) \\
47(63.5) \\
\end{array}$ & $\begin{array}{l}24(37.5) \\
40(62.5) \\
\end{array}$ & $\begin{array}{l}20(39.2) \\
31(60.8) \\
\end{array}$ & $\begin{array}{l}171(40.7) \\
249(59.3) \\
\end{array}$ & 0.681 \\
\hline $\begin{array}{l}\text { Due to your diet } \\
\text { have you ever } \\
\text { had any } \\
\text { nutritional }\end{array}$ & $\begin{array}{l}97(42) \\
134(58)\end{array}$ & $\begin{array}{l}30(40.5) \\
44(59.5)\end{array}$ & $\begin{array}{l}25(39.1) \\
39(60.9)\end{array}$ & $\begin{array}{l}24(47.1) \\
27(52.9)\end{array}$ & $\begin{array}{l}176(42) \\
244(58)\end{array}$ & 0.843 \\
\hline
\end{tabular}

Kurdistan Journal of Applied Research $\mid 4^{\text {th }}$ International Conference on the Health and Medical Science: Medical Researches Improve Life Quality (ICHMS 2020) | 57 


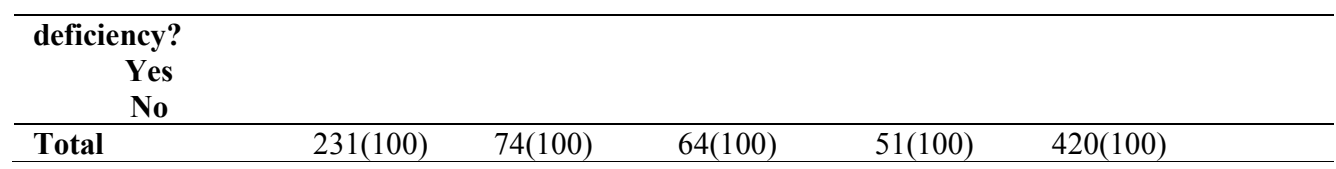

(Figure 4) shows that this bar chart shows the associations among colleges regarding practice of sleep with their lifestyle schedule and most of students $(59.3 \%)$ did not get enough sleep with their lifestyle, with highest percentage (71.6\%) from college of dentistry, $\mathrm{P}$ value of (0.001) showing significant association.

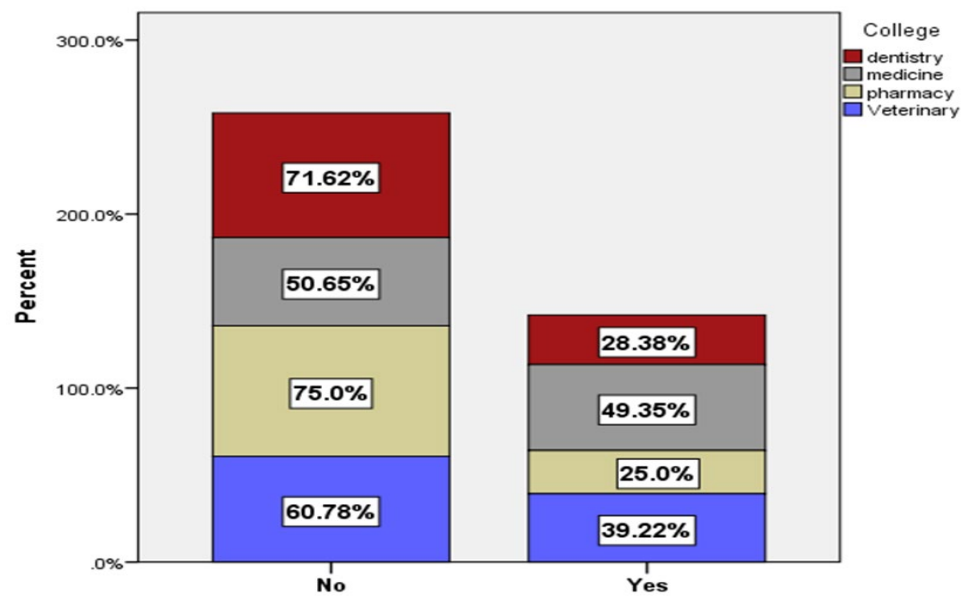

Figure 4: Associations among colleges students regarding if they get enough sleep with their lifestyle schedule.

\section{DISCUSSION}

In this study which was conducted among medical students in university of Sulaimani. Mean age of the participants were $20.88+/-1.482$ nearly similar to a research which was done in Malaysia [6]. This similarity is due to the standard age acceptable for medical college students. It was found that majority of the participants were females $(60.2 \%)$ this goes in contrast with a study done in India which number of male participants were more. [7] However this study is similar to a research done in Malaysia which female participants were more [6] and the explanation is that females were more cooperative for our participation than males. The majority of the responders were from inside city $(68.3 \%)$ this is similar to a study done in China [8] this is because that the resources for a modern lifestyle are more available in the city centers, and thereby they were more familiar with the topic and more cooperative. Most of the participants were among college of medicine $(55 \%)$ similar to a study done in Kuwait [9] Regarding the marital status nearly (93.3\%) of the responders were singles similar to a research done in Malaysia [6], this is because our participants are college students and were not at the appropriate age for commitment yet. Socioeconomic status of most of the students $(77.9 \%)$ was moderate similar to a research was done in Malaysia [6] Majority of the participants (75.95) were in normal Body Mass Index (BMI) group, this is similar to a research in Saudi Arabia [10] and this is because participants are college students and at a young age, with more care regarding their weight and appearance. Majority of the students $(82.1 \%)$ heard about modern lifestyle and they were mostly from college of medicine $(90.5 \%)$ with a significant association regarding P-value (0.001)in contrast to a research done in Kedah/Perlis among university students [11] it was found that the respondents knowledge of modern lifestyle was (52.1\%) and this difference has happened because medical college students have higher IQ and are usually from higher social classes and try to keep up to date 
with new changes. In this research, most of students (49.3\%) heard about it from internet with college of medicine taking highest percentage $(60.6 \%)$ with a significant association regarding $\mathrm{P}$ value (0.001). Most of students $(77.4 \%)$ knew that lifestyle can affect mental health, with highest percentage from college of medicine $(87 \%)$ with a significant association regarding $\mathrm{P}$ value (0.001), similar results were found in two researches, one done in Bochum, Germany, [12] another in Ohio state university. [13] And most of our participants knew that lifestyle can affect physical health $(78.3 \%)$ and they were mostly from college of medicine $(90.5 \%)$ with a significant association regarding $\mathrm{P}$ value $(0.001)$, in contrast, a study done in Saudi Arabia, king Saud university [10] students didn't largely relate lifestyle activities to have a great impact on health. In this study $(73.3 \%)$ of students knew that lifestyle choice to have impact on academic performance and they were mostly from college of medicine $(84 \%)$ with a significant association regarding $\mathrm{P}$ value $(0.001)$, this goes with a research done in Shahrekord university of medical sciences, Iran [14] this result is probably because all medical colleges have a great deal of requirements and a modern lifestyle can sometimes be an obstacle to a perfect academic performance. In this study (69.3\%) of students knew that technology has a major role in lifestyle, college medicine again taking highest percentage $(78.8 \%)$ with a significant association regarding $\mathrm{P}$ value $(0.001)$, this goes with a study done by American Heart Association.[15] About (68.3\%) of the students knew that healthy diet and medical checkup are a part of modern lifestyle, and college of medicine taking highest percentage (78.8\%) with a significant association regarding $\mathrm{P}$ value $(0.001)$ this goes in contrast to a study done in Kuwait university[16]

Regarding attitude, More than half of the participants $(54.5 \%)$ thought they are living in a healthy lifestyle and with a highest percentage in a college of dentistry $(66.2 \%)$, in contrast to a study was done in Spain University [17] which nearly half (47.7\%) of students didn't think they have a healthy lifestyle. Most of the participants $(64.3 \%)$ thought sometime changing their lifestyle inspired by other people, with highest percentage in college of medicine $(73.6 \%)$ with a significant association regarding $\mathrm{P}$ value $(0.001)$, this may be because of the influence of social media and surrounding people. Most of the participants (65.2\%) thought that sometimes they have been stressed due to their lifestyle with highest percentage in college of medicine $(70.6 \%)$, this goes in contrast to a research done in James Madison University ,Virginia ,USA [18] Which most of the students (50.8\%) thought that they always have been stressed due to their lifestyle this may be explained by their busy lifestyle due to colleges assignments and duties. About (66\%)of the students thought sometimes people pretend to have a certain lifestyle to attract others with a highest percentage in college of medicine $(75.3 \%)$ with a significant association regarding $\mathrm{P}$ value $(0.001)$, in contrast to a research done in university of north Florida, USA [19] because in our society our students care about other people's opinion. Almost half of the participants (49.5\%) thought financial status sometimes affects living a healthy lifestyle with highest percentage in college of medicine (57.6\%), which is similar to a research conducted in James Madison University, Virginia, USA [18] . Most of the participants (48.3\%) thought sometimes cultures have role in living a certain lifestyle with a highest percentage in college of veterinary $(56.9 \%)$ with a significant association regarding $\mathrm{P}$ value $(0.001)$, which is similar to a study done in University of India [20] because of the culture is conservative and restrictive in both societies. Most of the participants $(45.5 \%)$ thought sometimes religion have role on living a certain lifestyle with highest percentage in college of veterinary $(52.9 \%)$ with a significant association regarding $\mathrm{P}$ value (0.019), this goes in contrast with a study done in Putra University in Malaysia [21] where most of the students (52\%) thought religion always has a role on living a modern lifestyle because of influence of religion on relation with family and friends and basis of daily activity. Most of our participants (46.4\%) thought that geographical area always can affect lifestyle choice, with highest percentage in college of pharmacy $(51.6 \%)$, which is similarly an article by the earth bound reports [22] explains that geographical area and factors like climate and location is largely related to development, and this result may be due factors like war, poor economics and politics. Most of respondents in our study (64.5\%) thought that sometimes diet restriction due to certain lifestyle lead to nutritional deficiency with highest 
percentage in college of pharmacy (73.4\%), In contrast to a study done by Cleveland clinic [23] shows that students thought that always lifestyle choices with physical activity, poor diet and other choices can largely cause nutritional deficiency and chronic diseases like diabetes mellitus and hypertension.

Regarding practice, more than a half $(59.8 \%)$ of respondents with highest percentage $(62.3 \%)$ in medicine, are more stressed with their current life compared to their past, similarly a study done in university of Putra, Malaysia [24] this may be due to students did not fully understand their academic courses and get poor result for the subjects, and intrapersonal aspect is the concern about own appearance. About $63.8 \%$ had difficulty arranging time due to lifestyle with highest percentage being from college of medicine $(73.6 \%)$ with a significant association regarding $\mathrm{P}$ value (0.001) . Similarly, a research done in Dhofar university, Salalah, Oman.[25] this result is expected and may be due to lack of proper planning exam times and deadline projects. Majority of participants in our study (95\%) use social media with most of them being from college of medicine (97.4\%), compared to a study done in Dhofar university, Salalah, Oman. [25] similar response were found this may be due to the role and necessity of social media in college life such as keeping up with assignments and homework. Participants mostly $(70.9 \%)$ didn't play video games with highest percentage of respondents from college of medicine $(70.6 \%)$ in contrast, a study done in university of Cumberland, Kentucky, USA [26] about $(66 \%)$ of their student participants were online players, this difference is probably due to that our participants are from medical colleges and they have more assignment dues and many studies that can prevent them to include video games in their lifestyle. Highest percentage of our participants $(66 \%)$ didn't exercise and most of respondents were from college of Pharmacy (78.1\%) with a significant association regarding $\mathrm{P}$ value $(0.007)$, a similar result was found in a study done by college of education in Kuwait university, kaifan, Kuwait [16] this result may be due to lack of time, motivation, equipment. In this study most of students $(51.7 \%)$ didn't have depression because of not having a certain lifestyle, most of who responded were from college of pharmacy $(70.3 \%)$ with a significant association regarding $\mathrm{P}$ value $(0.003)$ in contrast to a study done in USA [27], this may be due to cultural and geographical differences. In our research, about half of the students (59.3\%) didn't get enough sleep with their lifestyle schedule, mostly being from college of pharmacy $(75 \%)$ with a significant association regarding $\mathrm{P}$ value (0.001), similar results were found in a study done in Kuwait university. [16] this result may be due to the stress and anxiety that mostly accompanies college lifestyle. Most of students in this study (59.3\%) didn't have a healthy diet with most of participants from college of dentistry $(63.5 \%)$ in contrast to a study done among university students [28] shows that most of their participants follow a healthy diet, this difference may be due to lack of nutritional knowledge and better and easy access to fast food during college hours and also simply not having time to cook a proper healthy meal. Most of participants (58\%) with most of them being from college of pharmacy (60.9\%) said that they didn't have any nutritional deficiency, but in contrast to a study done among university students in Australia [29] states that they got nutritional deficiencies and take supplements, this difference may be explained by persistent medical checkup in Australia where simplest nutritional deficiency can be detected.

\section{CONCLUSION}

Participants in general had knowledge and positive attitude regarding the topic but they had a negative practice. College of medicine had the highest knowledge regarding modern lifestyle, as most of the students were familiar with the topic and heard about it from internet. They had good knowledge regarding the aspects of our lives that are affected by our lifestyle such as mental/physical health and academic performance. They also acknowledged that technology has a major role in modern lifestyle. In addition that healthy diet and medical checkups also play a part, and in fact that illness or nutritional deficiencies can be related to an unhealthy lifestyle. Most participants from college of medicine believed that they are living a modern lifestyle, however keeping up with this lifestyle is stressful, and that some people pretend to 
have a certain lifestyle to attract others. They also believed that culture, religion, geographical location, and financial status can limit lifestyle choices. Majority of participants from college of pharmacy had a negative practice with modern lifestyle, in which they were feeling more stressed, used social media, did not exercise, were not on a healthy diet, and did not get enough sleep.

\section{REFERENCE}

[1] W. Inc. (2019). BD dictionary. Available: http://www.businessdictionary.com/definition/lifestyle.html

[2] A. study, "English Essay on "Modern Lifestyle" English Essay-Paragraph-Speech " 2019.

[3] D. D. Farhud, "Impact of lifestyle on health. Iran J Public Health," p. 44, 2015.

[4] C. G. Corina D.C, Geta M. ,Doina C.C D.C, "The importance of healthy lifestyle in modern society a medical, social, and spiritual perspective.," European Journal of Science and Theology, 2014.

[5] A. M, D. Z, D. M, and V. V, "Knowledge and Practice of Medical Students on Healthy Lifestyle: A CrossSectional Study in Shiraz," J Health Sci Surveillance Sys vol. 1, p. 80, 2013.

[6] R. A., Y. V. B., and N. A.B., "Practice among Malaysian University Students, Asian pacific journal of cancer prevention," 14, vol. 3, 2013.

[7] Shivaprasad and D. S. Prakash, "Modern lifestyle and its impact on well being of B- School students: A case study of AIMS, Bangalore. ," International Journal of Physical Education, Sports and Health 2016.

[8] D. Wang, X.-H. Xing, and X.-B. Wu, "Healthy Lifestyles of University Students in China and Influential Factors," Scientific world journal, 2013.

[9] D. A. Ali, "Knowledge of the Relationships between Oral Health, Diabetes, Body Mass Index and Lifestyle among Students at the Kuwait " University Health Sciences Center, Kuwait. Karger AG, 2015.

[10] K. M., W. B., J. M., T. H., M. Ali., and A. A., "Health promoting lifestyle of university students in saudi arabia," J BMC public Health vol. 18, 2018.

[11] N. i. Ahmad, M. R. Hamzah, and S. H. Ayub, "The Level od Knowledge,Attitude and Practice of Healthy Lifestyle among the University students in Kedah/Perlis," International Journal of Business and Techonopreneurship, vol. 1, no. 3, 2011.

[12] J. Velten, A. Bieda, S. Scholten, A. Wannemüller, and J. Margraf, "Lifestyle choices and mental health," BMC Public Health, 2018.

[13] B. M. Melnyk, C. Slevin, J. Hoying, and C. M. Alice Teall, "Physical health, lifestyle beliefs and behaviors, and mental healthof entering graduate health professional students," Journal of the American Association of Nurse practitioners, vol. 28, p. 8, 2015.

[14] M. Heidari, M. Borjian, M. Ghodusi, and M. Shirvani, "Relationship of lifestyle with academic achievement in Nursing students. ," Journal of clinical \& diagnostic research, vol. 11, p. 3, 2017.

[15] A. Afshin and D. mozaffarian, "Infornation technology and lifestyle," Journal of the American Heart Association, vol. 5, 2016.

[16] H. Ghaleb and N. Al-Khamees, "The Perception of College Students about a Healthy Lifestyle and its Effect on their Health.," Journal of Nutrition \& Food Sciences, vol. 5, p. 4, 2015.

[17] Veronica-varela, Jose.M, C. Ayan, V. Martin, and A. Molina, "Lifestyle and Health among Spanish University Students: Differences by Gender and Academic Discipline," International journal of environmental and public health, vol. 10, p. 8, 2012.

[18] J. Britz and E. Pappas, "Sources and Outlets of Stress among University Students: Correlations between Stress and Unhealthy Habits,"Undergraduate Research Journal For Human Sciences, 2010.

[19] T. L. Wright, "Body Image and Healthy Lifestyle Behavior Among University Students," no. University of North Florida. Florida, USA, 2012.

[20] A. Khare, "Impact of Indian Cultural Values and Lifestyles on Meaning of Branded Products: Study on University Students in India.," Journal Of International Consumer Marketing, vol. 23, no. 5, 2011.

[21] D. G. M. Baloch, A. R. Chhachhar, and W. V. Singutt, "Influence of Religion on Life Style and Social Behavior in Christianity Perspective of Malaysian Christian Student," Journal Of Basic And Applied Scientific Research, 2014.

[22] J. Williams. Geographical factors that affect development. Available: https://earthbound.report/2007/07/01/geographical-factors-that-affect-development/

[23] C. C. Foundation, "Lifestyle Choices: Root Causes of Chronic Diseases," 2013.

[24] H. Elias, W. S. Ping, and M. C. Abdullah, "Stress and Academic Achievement among Undergraduate Students in University Putra, Malaysia," Elsevier Ltd, 2011.

[25] S. Bashir, Z. Bashir, and M. Nazir, "Time Management and its Application in Academic Life: A Study of Omani Students at Dhofar University, Sultanate of Oman," Anglisticum Journal (IJLLIS), vol. 4, no. $2,2015$.

[26] J. Wright, "The effect of video game play on academic performance," Modern psychological studies, vol. 17, no. $1,2011$.

[27] C. H. van, G. I.J.M., H. Bosma, M. P. J. van, J. Jolles, and J. T.M., "Associations Between Lifestyle and Depressed Mood: Longitudinal Results From the Maastricht Aging Study," American Journal of Public Health, vol. 97, no. 5, 2007.

[28] N. Yahia, C. A. Brown, M. Rapley, and M. Chung., "Level of nutrition knowledge and its association with fat consumption among college students.," BMC Public Health, vol. 16, no. 1047, 2016.

[29] A. R., A.O.B.,G. K.,S. Cicerale , K.E., and M.G "Micronutrient Supplement Use and Diet Quality in

Kurdistan Journal of Applied Research $\mid 4^{\text {th }}$ International Conference on the Health and Medical Science: Medical Researches Improve Life Quality (ICHMS 2020) | 61 
University Students.," Nutrients journal, 2015.

Kurdistan Journal of Applied Research $\mid 4^{\text {th }}$ International Conference on the Health and Medical Science: Medical Researches Improve Life Quality (ICHMS 2020) $\mid 62$ 